\title{
BMJ Open Delivery room interventions to prevent bronchopulmonary dysplasia in preterm infants: a protocol for a systematic review and network meta-analysis
}

\author{
Souvik Mitra, ${ }^{1}$ Timothy Disher, ${ }^{2}$ Gerhard Pichler, ${ }^{3}$ Brandon D'Souza, ${ }^{4}$ \\ Helen Mccord, ${ }^{1}$ Varsha Chayapathi, ${ }^{1}$ Karlee Jones, ${ }^{1}$ Georg Schmölzer ${ }^{5,6}$
}

To cite: Mitra S, Disher T, Pichler G, et al. Delivery room interventions to prevent bronchopulmonary dysplasia in preterm infants: a protocol for a systematic review and network meta-analysis. BMJ Open 2019;9:e028066. doi:10.1136/ bmjopen-2018-028066

- Prepublication history and additional material for this paper are available online. To view please visit the journal (http:// dx.doi.org/10.1136/bmjopen2018-028066).

Received 21 November 2018 Revised 07 June 2019 Accepted 15 July 2019

Check for updates

(C) Author(s) (or their employer(s)) 2019. Re-use permitted under CC BY-NC. No commercial re-use. See rights and permissions. Published by BMJ.

For numbered affiliations see end of article.

Correspondence to Dr Georg Schmölzer; georg.schmoelzer@me.com

\section{ABSTRACT}

Introduction As gestational age decreases, incidence of bronchopulmonary dysplasia (BPD) and chronic lung disease increases. There are many interventions used in the delivery room to prevent acute lung injury and consequently BPD in these patients. The availability of different treatment options often poses a practical challenge to the practicing neonatologist when it comes to making an evidence-based choice as the multitude of pairwise systematic reviews including Cochrane reviews that are currently available only provide a narrow perspective through head-to-head comparisons. Methods and analysis We will conduct a systematic review of all randomised controlled trials evaluating delivery room interventions within the first golden hour after birth for prevention of BPD. The primary outcome includes BPD. Secondary outcomes include death at 36 weeks of postmenstrual age or before discharge; severe intraventricular haemorrhage (grade 3 or 4 based on the Papile criteria); any air leak syndromes (including pneumothorax or pulmonary interstitial emphysema); retinopathy of prematurity (any stage) and neurodevelopmental impairment at 18-24 months. We will search from their inception to August 2018, the following databases: Medline, EMBASE and Cochrane Central Register of Controlled Trials as well as grey literature resources. Two reviewers will independently screen titles and abstracts, review full texts, extract information and assess the risk of bias and the confidence in the estimate (with Grading of Recommendations Assessment, Development and Evaluation approach). This review will use Bayesian network meta-analysis approach which allows the comparison of the multiple delivery room interventions for prevention of BPD. We will perform a Bayesian network meta-analysis to combine the pooled direct and indirect treatment effect estimates for each outcome, effectiveness and safety of delivery room interventions for prevention of BPD.

Ethics and dissemination The proposed protocol is a network meta-analysis, which has been registered on PROSPERO International prospective register of systematic reviews (CRD42018078648). The results will provide an evidence-based guide to choosing the right sequence of early postnatal interventions that will be associated with the least likelihood of inducing lung injury and BPD in preterm infants. Furthermore, we will identify
Strengths and limitations of this study

- Comprehensive search to include published randomised clinical trials in the most important databases, as well as unpublished work.

- Use the novel method for rating the confidence in the estimates recommended by the Grading of Recommendations Assessment, Development and Evaluation working group.

- We will employ a novel Stochastic multicriteria acceptability analysis model to determine the most effective sequence of delivery room interventions with respect to the most important clinical outcomes.

- We anticipate some degree of clinical heterogeneity while considering such a large number of competing and non-competing delivery room interventions.

- Potential to lump interventions into single nodes or split a node into multiple nodes to generate clinically meaningful results

knowledge gaps and will encourage further research for other therapeutic options. Therefore, its results will be disseminated through peer-reviewed publications and conference presentations. Due to the nature of the design, no ethics approval is necessary.

\section{INTRODUCTION}

Bronchopulmonary dysplasia (BPD) is the most common chronic respiratory morbidity associated with premature birth. BPD is defined as either need of supplemental oxygen at (i) 28 days postnatal age ${ }^{1}$ or (ii) 36 weeks of postmenstrual age with or without compatible clinical and radiographic findings. ${ }^{2}$ BPD affects $30 \%-60 \%$ of prematurely born infants with the incidence being inversely proportional to gestational age. ${ }^{3}$ With increased survival of extremely low gestational age newborns (ELGAN), the incidence of BPD continues to increase despite improvement in neonatal care practices over the last two decades. ${ }^{3} \mathrm{BPD}$ is known to be associated with long-term respiratory 


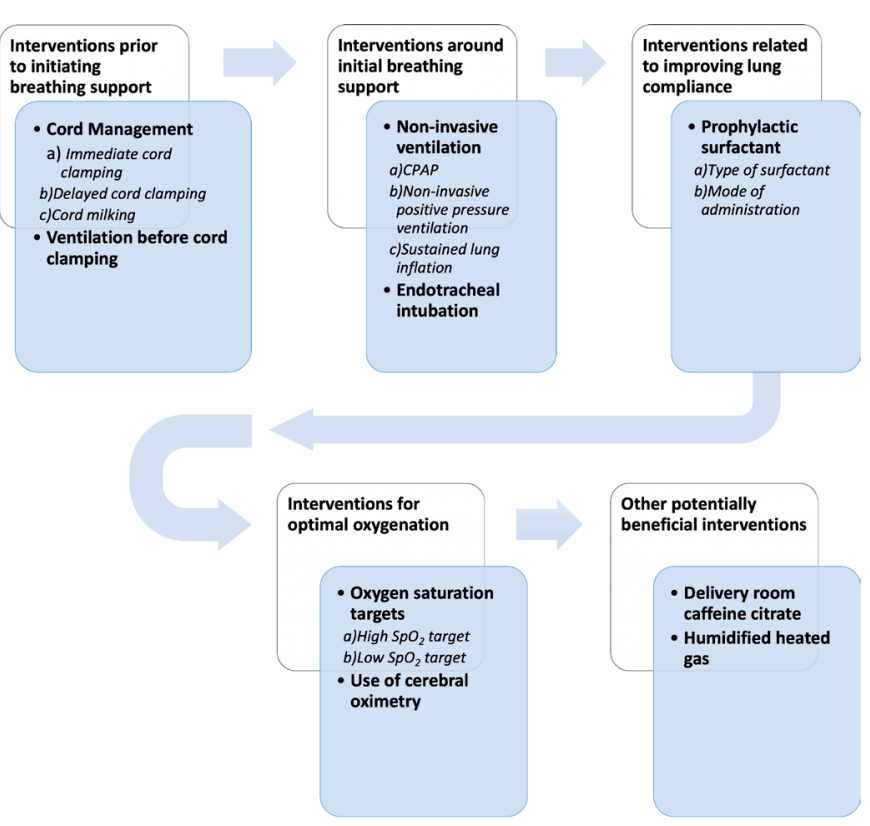

CPAP: Continuous Positive Airway Pressure; $\mathrm{SpO}_{2}$ : Oxygen saturation

Figure 1 Physiological sequence of potential delivery room interventions.

morbidity that persists into adolescence and adulthood. ${ }^{45}$ There is also increasing evidence that BPD and duration on supplemental oxygen have long-term adverse effects on cognitive and academic achievement with each per cent increase in BPD rate being associated with a $0.01 \mathrm{SD}$ decrease in IQ $(0.15 \mathrm{IQ}$ points $)(\mathrm{p}<0.001) .{ }^{67}$

Several antenatal, perinatal and postnatal factors contribute to the development of BPD. It is postulated that early lung injury and inflammation play an important role in the pathogenesis of BPD. ${ }^{89}$ In the fetus, the gas exchange organ is the placenta and the function of gas exchange is transferred from the placenta to the lungs immediately after birth. Therefore, the newborn infant's lungs must open and be aerated to allow the transition from fetal to postnatal circulation and physiology. However, in ELGANs, several physiological factors prevent this transition. These include lack of surfactant leading to increased alveolar surface tension, non-compliant chest wall and weak respiratory muscles. ${ }^{10-12}$ Therefore, most ELGANs require assisted ventilation and/or supplemental oxygen after birth to ensure optimal gas exchange. However, both therapies may also induce lung inflammation due to barotrauma and/or volutrauma and oxygen-free radical generation, thereby initiating the pathogenesis of BPD. Therefore, any interventions targeted at limiting lung injury and oxidative stress during resuscitation in the delivery room immediately after the birth may help to prevent the development of BPD or reduce its severity.

A number of clinical trials have been conducted on a variety of delivery room interventions, including (i) interventions prior to initiating breathing support (ie, clamping vs milking the umbilical cord); (ii) interventions around initial breathing support (ie, continuous positive airway pressure (CPAP), non-invasive positive pressure ventilation, sustained lung inflation or endotracheal intubation); (iii) interventions related to improving lung compliance (ie, prophylactic surfactant therapy including the different variations in its administration modalities); (iv) interventions related to minimising oxidative stress (ie, higher vs lower oxygen saturation targets), (v) use of cerebral oximetry and (vi) other potentially beneficial therapies such as caffeine administration (figure 1). ${ }^{13} 14$

The availability of multiple potential interventions in a resuscitation scenario often poses a practical challenge to healthcare professionals as to which sequence of interventions would provide the greatest likelihood of minimising BPD and which interventions are unnecessary and unlikely to be of any benefit. ${ }^{13}$ There have been previous systematic reviews and pairwise meta-analyses on the different competing interventions such as initial breathing support and oxygen saturation targets. ${ }^{15-17}$ However, these meta-analyses, though well conducted, provide a narrow perspective to the situation where a sequence of non-competing interventions occur within a short time frame, whereas each intervention has potentially competing variations. The use of a network meta-analysis (NMA) framework may help to provide a more feasible, comprehensive and evidence-based solution to the dilemma that healthcare professionals face during resuscitation of ELGANs with regard to multiple competing interventions aimed at mitigating lung injury. The Cochrane handbook considers NMA as a highly valuable tool to evaluate and rank treatment options according to their safety and effectiveness. ${ }^{18}$ Bayesian NMAs have been proposed as an effective method for evaluating the effectiveness of multiple competing interventions. ${ }^{18-20}$ Delivery room interventions consist of a sequence of non-competing category of interventions and within each category there are several potentially competing interventions (figure 1). Given that many of these competing delivery room interventions have not been compared in head-to-head studies, we expect that some of the possible comparisons between the interventions will not have direct evidence. Hence, we will perform a random effects (RE) NMA. Delivery room interventions will be defined as all potential interventions in the immediate postnatal period. $^{21-23}$

\section{Objectives}

To determine the relative effectiveness of commonly practiced delivery room interventions for preterm infants born $<33$ weeks of gestation in preventing BPD using a Bayesian NMA.

\section{METHODS AND DESIGN}

This systematic review and NMA protocol have been registered on PROSPERO (International prospective register of systematic reviews) (CRD42018078648). This protocol was developed following the Preferred Reporting Items for Systematic Review and Meta-Analysis Protocols 
(PRISMA-P) guidance. ${ }^{24}$ The final report will comply with the recommendations of the PRISMA Extension Statement for Reporting of Systematic Reviews Incorporating Network Meta-analyses of Healthcare Interventions. ${ }^{25}$

\section{Search strategy}

We will search from their inception to August 2018, the following databases: MEDLINE, EMBASE and the Cochrane Central Register of Controlled Trials. We will use combination of controlled terms (Medical Subject Heading, MeSH and Emtree), and free-text terms with various synonyms for the different possible delivery room interventions and BPD. Search alerts will be set up for monthly notification and the search will be repeated before the final manuscript submission to identify any new relevant trials. Search strategies have been developed with liaison with an experienced librarian. No language, publication status or date limit will be used. The search strategies have been detailed in online supplementary appendix A.

We will seek registered details of selected trials in the US National Institutes of Health resource (www.clinicaltrials.gov) and the WHO International Clinical Trials Registry Platform Search Portal. We intend to obtain additional grey literature from personal communication from experts in the field, reviewing the reference lists of relevant articles, abstracts and conference proceedings (Society for Paediatric Research, European Society for Paediatric Research) and seeking results of unpublished trials. We intend to contact authors of unpublished work and authors of published trials to clarify information that is not clear in the articles.

\section{Eligibility criteria}

We will include randomised controlled trials that evaluate the effectiveness of commonly practiced delivery room interventions. Studies will have to have the following characteristics regarding participants, intervention, control and type of study.

a. Participants: Preterm infants ( $<33$ weeks) requiring intervention(s) during neonatal transition within the first golden hour after birth.

b. Interventions include the following: (i) cord management (including immediate cord clamping, delayed cord clamping, cord milking and/or resuscitation attached to the cord); (ii) respiratory support (including positive pressure ventilation, CPAP, sustained lung inflation and/or intubation, and mechanical ventilation); (iii) surfactant delivery (type of surfactant delivered via endotracheal tube or laryngeal mask, intubate-surfactant-extubate technique, less-invasive surfactant administration/minimally invasive surfactant therapy and/or nebulised surfactant administration; (iv) initial fractional concentration of inspired oxygen ( $\leq 0.3$ or $\geq 0.6$ ); (v) monitoring during resuscitation (respiratory function monitor, near-infrared spectroscopy); (vi) medication (eg, caffeine citrate or diuretics) and (vii) use of heated, humidified gas c. Comparator: One or more of the above interventions compared with each other or no treatment.

Since interventions possible in a delivery room are largely related to delivery room resources, we have a priori decided to only include interventions that the infants were subjected to in the immediate postnatal period irrespective of whether these interventions were physically carried out in the room where the infant was born. Studies that examined interventions that were carried out after the initial stabilisation period (no randomisation within the first hour after birth, for example, feeding, indomethacin, antibiotics) will be excluded from the review. Furthermore, studies must have randomised within the first hour after birth to be eligible. This approach potentially will include studies with randomisation within the first hour after birth but study intervention administration within the first 2 hours after birth (eg, surfactant or caffeine administration).

\section{Outcomes}

Our primary outcome is BPD (defined as oxygen requirement at 36 weeks of postmenstrual age). Secondary outcomes include death at 36 weeks of postmenstrual age or before discharge; severe intraventricular haemorrhage (grade 3 or 4 based on the Papile criteria) ${ }^{26}$; any air leak syndromes (including pneumothorax or pulmonary interstitial emphysema); retinopathy of prematurity (any stage) and neurodevelopmental impairment at 18-24 months. All the outcomes, its definitions and measures are detailed in table 1 . We aim to perform one subgroup analysis comparing infants $<28$ weeks ver $29-32$ weeks.

\section{Patient and public involvement}

Neither patients nor public were involved in the development of the research question or design of this study. This NMA does not recruit any patients. The study will be published and presented at conferences to healthcare professionals.

\section{Study selection}

The titles and abstracts retrieved will be screened by two independent reviewers in duplicate to assess its eligibility using the Covidence platform. ${ }^{27}$ As a second step, the full-text articles of the potentially eligible studies will be screened to assess their eligibility. We will include the full text of all studies for which both reviewers agree about their inclusion. For both steps, any disagreements between the reviewers will be resolved by discussion and if no agreement can be reached, a third member of the team will decide whether the study shall be included or not. We will refer to inclusion and exclusion criteria during the screening process. Records of ineligible fulltext articles along with the reason for ineligibility will be saved for future reference. Eligible articles citations will be uploaded to Covidence. We will present the PRISMA flow diagram ${ }^{28}$ demonstrating the search and screening process. 
Table 1 A priori defined outcome measures

\begin{tabular}{ll}
\hline Outcome measure & Definition \\
\hline $\begin{array}{l}\text { Bronchopulmonary dysplasia } \\
\text { Mortality }\end{array}$ & $\begin{array}{l}\text { No. of neonates who require oxygen at } 36 \text { weeks of postmenstrual age } \\
\text { Death before discharge }\end{array}$ \\
$\begin{array}{l}\text { Severe intraventricular } \\
\text { haemorrhage }\end{array}$ & $\begin{array}{l}\text { No. of neonates with grades 3-4 based on the Papile criteria } \\
\text { Air leak syndromes }\end{array}$ \\
$\begin{array}{ll}\text { No. of neonates with pneumothorax or pulmonary interstitial emphysema confirmed by chest } \\
\text { X-ray }\end{array}$ \\
$\begin{array}{l}\text { Netage) } \\
\text { Neurodevelopmental impairment neonates with any stage of retinopathy of prematurity as per the international } \\
\text { classification of retinopathy of prematurity }\end{array}$ \\
$\begin{array}{l}\text { No. of infants with any degree of neurodevelopmental impairment as assessed by a } \\
\text { standardised and validated assessment tool, a child developmental specialist or both, at any } \\
\text { age reported (outcome data grouped at 12, } 18 \text { and } 24 \text { months if available) }\end{array}$ \\
\hline
\end{tabular}

\section{Data abstraction}

A pre-specified standardised data extraction form in a Microsoft Excel sheet will be used to extract the data from the eligible studies. The data extraction form will be pilot tested independently by all reviewers before its use, to standardise the process. Eight reviewers will carry out the extraction, working independently in pairs and in duplicate. In case of disagreement in assessing the methodological quality of the study, we will try to resolve it by consensus. If consensus cannot be reached, a third designated reviewer will be involved. We will contact authors of primary studies, during data extraction, to provide any missing information.

\section{Node formation}

Within each component of the stabilisation pathway, we anticipate the identification numerous similar non-competing interventions (eg, multiple synthetic and natural surfactants). In an iterative process, clinical experts (GS, SM and GP), blinded to the implications for effect estimates, will come to consensus on definitions of nodes and be presented with the implications of those decisions via network diagrams (eg, lumping causing the loss of trials comparing lumped interventions, splitting causing disconnected networks). Experts will then be asked to identify whether groups of treatments should be defined as classes (eg, natural vs synthetic surfactant) or lumped together. Class-based models have the advantage of offering an estimate of class effect as well as shrunken effects of individual treatments, while lumping can allow for more robust estimation of between-trial variability, and can reduce the probability of chance violations of NMA assumptions. This process will be repeated until a consensus decision is reached on the node making algorithm that meets the criteria of satisfying clinical demands, preserving the assumption that interventions within nodes are sufficiently similar and presenting data in the least aggregated form possible. ${ }^{29}$

\section{Assessment of risk of bias}

The risk of bias (ROB) of eligible studies will be assessed according to a modified version of the Cochrane
Collaboration's ROB tool. ${ }^{18}$ The six criteria to be assessed are as follows: sequence generation, allocation concealment, blinding of participants, personnel and outcome assessors, completeness of follow-up, selective outcome reporting, and presence of other biases. Each domain will be assigned a score 'definitely low risk' or 'definitely high risk' or 'unclear risk'. Two independent reviewers will assess the ROB. We will try to reach consensus when disagreements between two reviewers when assessing the methodological quality of the studies. Nevertheless, if consensus cannot be reached, a third reviewer will resolve it.

\section{Measures of treatment effect}

Effect estimates along with 95\% credible intervals (CrIs) will be estimated using risk ratios (RRs) calculated using methods described by Dias, using the baseline risk parameter to convert ORs to RRs. ${ }^{29}$ When RE models are used, estimates will also be accompanied by their $95 \%$ predictive interval representing the interval within which we would expect the treatment effect of a future study to lie. ${ }^{30}$ Relative treatment rankings will be summarised using mean ranks with their 95\% CrIs and the surface under the cumulative ranking curve (SUCRA) values and cumulative probability rankograms. ${ }^{32}$ SUCRAs range from $0 \%$ to $100 \%$ with values of $100 \%$ representing a hypothetical treatment that is always best without uncertainty.

\section{Assessment of reporting bias}

We will construct a comparison adjusted funnel plot for the network to assess the potential publication bias and small-study effects, ${ }^{33}$ if we retrieve at least 10 studies. We will inspect plots visually for evidence of asymmetry and if publication bias is suspected, we will conduct a sensitivity analysis using models described by Mavridis, Welton and Salanti. ${ }^{34}$

\section{Assessment of transitivity assumption}

Clinical experts (GS, GP and SM) will assess trial characteristics using tables and visualisations to assess whether the transitivity assumption is likely to hold. The characteristics to be evaluated are those that are expected to be 
effect modifiers and will include gestational age, birth weight, baseline event risk and ROB. These assessments will be made prior to any meta-analysis to limit to influence of presence or absence of statistical heterogeneity on the assessment of the transitivity assumption. The decision to pool data will be based on consensus. If it is determined that quantitative synthesis is inappropriate, we will summarise our findings narratively.

\section{Direct treatment comparisons}

Given that we expect clinical and methodological heterogeneity among the studies (see below in Rating the Confidence in Estimates section), which, in turn, will create statistical heterogeneity, we will pool evidence for each treatment comparison using a Bayesian RE model. ${ }^{35}$ In comparison to the fixed-effect model (FE), the RE model is conservative in the sense that it accounts for both within-study and between-study variability. The RE model assumes that the observed treatment effect for a study is a combination of a treatment effect common to all studies plus a component specific to that study alone ${ }^{3637}$ Models will be based on standard code modified to include minimally informative priors on baselines, treatment effects and between trial heterogeneity. ${ }^{38}$ These priors generally provide more stable estimates, particularly in cases where data are sparse, will be developed using the approach described by Gabry et al. ${ }^{39} 40$

\section{Network meta-analysis}

For each outcome, we will present the network diagram and a forest plot compared against the common comparator with the network estimates as well as league tables showing all pairwise comparisons. To capture the non-competing nature of interventions along the stabilisation pathway and to directly fulfil the research objectives, we will use component models as described by Welton et $\mathrm{al}^{41}$ These models assume that interventions across domains of the stabilisation pathway are additive on additive on logit scale for dichotomous outcomes (eg, mortality). In the absence of direct evidence for a given comparison, an indirect comparison will provide an estimate of the treatment effect. In the presence of direct evidence, the NMA will provide a combined estimate (ie, direct and indirect evidence) ${ }^{32}$ For instance, in a triangular network $\mathrm{ABC}$ composed by studies that directly compare A versus B and A versus $\mathrm{C}$ treatments, we can indirectly estimate the effect of B versus $\mathrm{C}$ treatments. In case direct evidence of $\mathrm{B}$ versus $\mathrm{C}$ treatment comparison is also available, then a combined estimate of direct and indirect evidence of $\mathrm{B}$ versus $\mathrm{C}$ can be calculated using a NMA.

We will fit a Bayesian hierarchical model with weakly informative priors (ie, normal with mean zero and SD 5 for outcomes on the logit scale) adjusting for correlation of multi-arm trials, and assuming a common-within network heterogeneity variance (uniform on $0-2$ ). We will assess heterogeneity by estimating the magnitude of the between-study variance. ${ }^{42}$ If the posterior estimate of between-study variance shows signs of prior dominance (eg, extreme values and long tails, ORs approaching infinity), we will assess whether using the empirically estimated informative prior distribution described by Turner et $a l^{43}$ provides more sensible estimates. If the network structure is such that estimates of CrIs are sufficiently different from original trial estimates and lack clinical validity, we will also present results from an FE model. In this case, we will caution against overinterpretation of CrIs. Markov chains will be run for a sufficient number of iterations to reach convergence, which we will assess on the basis of the Brooks-Gelman-Rubin diagnostic, with values less than 1.05 considered acceptable if consistent with visual inspection of convergence and time series plots. ${ }^{44}$ All analyses will be performed in JAGS or similar software via the statistical programme R. ${ }^{4546}$

\section{Assessment of inconsistency}

Inconsistency is the statistical manifestation of the violation of the transitivity assumption, which presents as a disagreement between direct and indirect estimates (loop inconsistency), and/or inconsistency between studies that inform the same treatment comparison, but include a different number of treatment arms (design inconsistency). To evaluate both design and loop inconsistency, we will apply the design-by-treatment interaction model with random inconsistency effects. ${ }^{48} 48$ These findings will be interpreted within the context of the estimate of between-trial variance as these concepts are closely related and difficult to separate. For example, large estimates of between-trial variance are indicative of heterogeneity within direct comparisons but may also be the result of inconsistency between direct and indirect evidence.

\section{Exploration of heterogeneity and inconsistency}

We will perform a network meta-regression using a potential effect modifiers to explore important heterogeneity and/or inconsistency. We propose the following potential sources of heterogeneity, which could be possible effect modifiers: gestational age, birth weight and ROB. We hypothesise that lower gestational age and low ROB will be related to less effectiveness of interventions. Overall, ROB will be determined by taking the average of the three most important ROB items identified by expert consensus (sequence generation, allocation concealment and blinding). Meta-regression models will assume a single shared coefficient for all non-baseline treatments. ${ }^{42}$ Interpretation of meta-regression models will be in keeping with suggestions from Dias et al, namely ${ }^{1}$ inclusion of the coefficient leads to a decrease in the estimate of between-study variance and ${ }^{2}$ the $95 \%$ CrIs of the estimated coefficient exclude the null. ${ }^{42}$

\section{Sensitivity analyses}

We plan to perform sensitivity analyses of different heterogeneity priors to assess the robustness of results. ${ }^{354349}$ Furthermore, findings from component models will be compared against a model without this assumption. In 
both cases, we will compare model fit using both absolute (residual deviance) and relative (DIC) measures as well as a qualitative assessment of whether the analysis leads to an important change in effect estimates.

\section{Rating the confidence in estimates of the effect}

We will assess the confidence in the estimates for each outcome using the Grading of Recommendations Assessment, Development and Evaluation (GRADE) approach. ${ }^{50}$ For this purpose, two authors will independently do the assessment. The confidence in the estimates will be based on four levels: high, moderate, low and very low. For the direct comparisons, we will assess and rate each outcome based on the categories: ROB imprecision, inconsistency and publication bias. ${ }^{51-55}$ To assist with assessment of each domain, we will use threshold plots, which show the smallest change in study-level/contrast-level estimates required to change the conclusions of the analysis.

We will assess and rate the confidence in all the indirect comparisons-if available-obtained from first-order loops (FOLs) following the GRADE categories used for assessing the direct comparisons in addition to the transitivity assessment. Transitivity, also called similarity ${ }^{56}$ is the assumption that an indirect comparison is a valid method to compare two treatments that have not been compared in a head-to-head trial because the studies are sufficiently similar in important clinical and methodological characteristics, or in other words, that they are similar in their distributions of effect modifiers. ${ }^{57} 58$ Then, we will rate the confidence in each NMA effect estimate using the higher rating when both direct and indirect evidence are present.

We will assess and rate the confidence in estimates of effect from the direct comparisons in our pairwise meta-analyses described previously. To rate the confidence in the indirect comparisons, we will focus our assessments on FOLs, that is, loops connected to the interventions of interest through only one other intervention. For instance, if for $\mathrm{A}, \mathrm{B}$ and $\mathrm{C}$ interventions, there are direct comparisons of $A$ versus $B(A B)$ and $B$ versus $\mathrm{C}(\mathrm{BC})$, we will be able to indirectly estimate the effects of $A$ versus $C(A C)$. The $A C$ indirect estimation will be a FOL. We will choose the FOLs with the lowest variances for rating the confidence as they contribute the most to the estimates of effect.

Within FOLs, the indirect comparison confidence will be the lowest of the confidence ratings we have assigned to the contributing direct comparisons. For example, if we find that $\mathrm{AB}$ has moderate confidence and $\mathrm{BC}$ has high confidence, we will judge the associated indirect comparison, AC, as moderate confidence. We may rate down confidence in the indirect comparisons further if we have a strong suspicion that the transitivity assumption has been violated.

Our overall judgement of confidence in the NMA estimate for any pairwise comparison will be the higher of the confidence rating among the contributing direct and indirect comparisons. However, we may rate down confidence in the network estimate if we find that the direct and indirect estimates have inconsistency. For this purpose, the GRADE approach recommends to assess the incoherence (or inconsistency as described in the 'Network Meta-Analysis' section) criteria, which is defined as the differences between direct and indirect estimates of effect. ${ }^{59}$

\section{Multicriteria acceptability analysis}

NMAs provide an estimate of effect estimates of competing interventions, but this alone is not sufficient to aid decision-making. We will aim to supplement this review with a stochastic multicriteria acceptability analysis (SMAA) using methods defined by Tervonen and Van Valkenhoef. ${ }^{60}$ These methods use a partial value function to allow for a quantitative risk-benefit analysis across multiple outcomes, given an ordinal ranking of importance for decision-making (eg, mortality $>\mathrm{BPD}$ ). Based on the best fitting NMA model, we will conduct SMAAs: one without preference criteria and a second with preference criteria determined by expert consensus. Since SMAA is based on estimates constrained to the interval $[0,1]$, we will calculate absolute risk of outcomes based on either a suitably designed observational trial or, if unavailable, a pooled control arm risk of included studies in the reference treatment. ${ }^{61}$ If treatments require the trade-off of common (eg, BPD) and very rare (eg, mortality) events, we will use the $95 \%$ CrI hull approach. ${ }^{62}$ The outputs of the SMAA will be a rankogram outlining the probability that a treatment is best, second best, etc, a vector of central weights, a confidence factor for the preference-free model; and the rankogram for the ordered model. The vector of central weights provides a summary of the implied preferences required to hold an a priori preference for one treatment or another (ie, the outcome preference implied by a clinician's current practice). The confidence factor is the probability that a treatment is best given these preferences, and is used as a measure of uncertainty.

\section{DISCUSSION}

Interventions in the immediate postnatal period may have long-term clinical implications. This NMA will provide the relative effectiveness of commonly practiced delivery room interventions for preterm infants born $<33$ weeks of gestation in preventing BPD. To the best of our knowledge, this will be the first review that will examine the relative effectiveness of each delivery room intervention individually and in combination with respect to important clinical outcomes using novel statistical techniques. Its results will be of interest for a broad range of audience: practice guideline developers, paediatricians, neonatologists, policymakers and researchers, as it could be used to provide clinical recommendations for the choice of sequence of delivery room interventions.

Our review will have several methodological strengths. First, we will implement a wide comprehensive search to include published randomised clinical trials in the most 
important databases, as well as unpublished work. Second, we will use the novel method for rating the confidence in the estimates recommended by the GRADE working group. Third, we will employ a novel SMAA model to determine the most effective sequence of delivery room interventions with respect to the most important clinical outcomes. ${ }^{60}$ On the other hand, we anticipate some methodological challenges while undertaking such a review. We anticipate some degree of clinical heterogeneity while considering such a large number of competing and non-competing delivery room interventions. Based on the number of interventions identified following the systematic review, we may have to lump interventions into single nodes or split a node into multiple nodes to generate clinically meaningful results.

We hope that this review will provide an evidencebased guide to choosing the right sequence of early postnatal interventions that will be associated with the least likelihood of inducing lung injury and BPD in preterm infants.

\section{Author affiliations}

${ }^{1}$ Department of Pediatrics, Dalhousie University, IWK Health Center, Halifax, Nova Scotia, Canada

${ }^{2}$ School of Nursing, Dalhousie University, Halifax, Nova Scotia, Canada

${ }^{3}$ Research Unit for Neonatal Micro- and Macrocirculation, Department of Pediatrics, Medical University of Graz, Graz, Austria

${ }^{4}$ Department of Respiratory Therapy, IWK Health Centre, Halifax, Nova Scotia, Canada

${ }^{5}$ Centre for the Studies of Asphyxia and Resuscitation, Neonatal Research Unit, Royal Alexandra Hospital, Edmonton, Alberta, Canada

${ }^{6}$ Department of Pediatrics, University of Alberta, Edmonton, Alberta, Canada

Contributors Conception and design: GS and SM; Data analysis plan: GMS, SM, GP, TD, BDS, HM, VC and KJ; Drafting of the article: GS, SM, GP, TD, BDS, HM, VC and $\mathrm{KJ}$; Critical revision of the article for important intellectual content: GS, SM, GP, TD, BDS, HM, VC and KJ; Final approval of the article: GS, SM, GP, TD, BDS, HM, VC and $\mathrm{KJ}$.

Funding We would like to thank the public for donating money to our funding agencies: GMS is a recipient of the Heart and Stroke Foundation/University of Alberta Professorship of Neonatal Resuscitation, a National New Investigator of the Heart and Stroke Foundation Canada and an Alberta New Investigator of the Heart and Stroke Foundation Alberta. This research has been facilitated by the Women and Children's Health Research Institute through the generous support of the Stollery Children's Hospital Foundation.

Competing interests TD provides methodological advice for Cornerstone Research Group Inc. Cornerstone Research Group Inc. consults for various pharmaceutical and medical device companies. BD has provided device training and educational presentations to clinicians on behalf of Mallinckrodt Pharmaceuticals. GS has registered the RETAIN neonatal resuscitation board (Tech ID 2017083) and RETAIN neonatal resuscitation video game (Tech ID 2017086) under Canadian copyright, and GS is the owner of RETAIN Labs Inc. (https://www.playretain.com), which is distributing these games.

Patient consent for publication Not required.

Ethics approval Final approval of the article: GS, SM, GP, TD, BDS, HM, VC, KJ.

Provenance and peer review Not commissioned; externally peer reviewed.

Open access This is an open access article distributed in accordance with the Creative Commons Attribution Non Commercial (CC BY-NC 4.0) license, which permits others to distribute, remix, adapt, build upon this work non-commercially, and license their derivative works on different terms, provided the original work is properly cited, appropriate credit is given, any changes made indicated, and the use is non-commercial. See: http:// creativecommons.org/licenses/by-nc/4.0/.

\section{REFERENCES}

1. Bancalari E, Abdenour GE, Feller R, et al. Bronchopulmonary dysplasia: clinical presentation. J Pediatr 1979;95:819-23.

2. Shennan AT, Dunn MS, Ohlsson A, et al. Abnormal pulmonary outcomes in premature infants: prediction from oxygen requirement in the neonatal period. Pediatrics 1988;82:527-32.

3. Stoll BJ, Hansen NI, Bell EF, et al. Eunice Kennedy Shriver National Institute of child health and human development neonatal research network. trends in care practices, morbidity, and mortality of extremely preterm neonates, 1993-2012. JAMA 2015;314:1039-51.

4. Doyle LW, Faber B, Callanan C, et al. Bronchopulmonary dysplasia in very low birth weight subjects and lung function in late adolescence. Pediatrics 2006;118:108-13.

5. Fawke J, Lum S, Kirkby J, et al. Lung function and respiratory symptoms at 11 years in children born extremely preterm: the EPICure study. Am J Respir Crit Care Med 2010;182:237-45.

6. Short EJ, Klein NK, Lewis BA, et al. Cognitive and academic consequences of bronchopulmonary dysplasia and very low birth weight: 8-year-old outcomes. Pediatrics 2003;112:e359.

7. Twilhaar ES, Wade RM, de Kieviet JF, et al. Cognitive outcomes of children born extremely or very preterm since the 1990s and associated risk factors: a meta-analysis and meta-regression. JAMA Pediatr 2018;172:361-7.

8. Hillman NH, Moss TJM, Kallapur SG, et al. Brief, large tidal volume ventilation initiates lung injury and a systemic response in fetal sheep. Am J Respir Crit Care Med 2007;176:575-81.

9. Wallace MJ, Probyn ME, Zahra VA, et al. Early biomarkers and potential mediators of ventilation-induced lung injury in very preterm lambs. Respir Res 2009;10:19.

10. Obladen M. Factors influencing surfactant composition in the newborn infant. Eur J Pediatr 1978;128:129-43.

11. Heldt GP, Mcllroy MB. Distortion of chest wall and work of diaphragm in preterm infants. J Appl Physiol 1987;62:164-9.

12. Heldt GP, Mcllroy MB. Dynamics of chest wall in preterm infants. $J$ Appl Physiol 1987;62:170-4.

13. Foglia EE, Jensen EA, Kirpalani H. Delivery room interventions to prevent bronchopulmonary dysplasia in extremely preterm infants. $J$ Perinatol 2017;37:1171-9.

14. Rich WD, Leone T, Finer NN. Delivery room intervention: improving the outcome. Clin Perinatol 2010;37:189-202.

15. Fogarty M, Osborn DA, Askie L, et al. Delayed vs early umbilical cord clamping for preterm infants: a systematic review and meta-analysis. Am J Obstet Gynecol 2018;218:1-18.

16. Schmölzer GM, Kumar M, Pichler G, et al. Non-Invasive versus invasive respiratory support in preterm infants at birth: systematic review and meta-analysis. BMJ 2013;347.

17. Pichler G, Urlesberger B, Baik N, Schwaberger B, et al. Cerebral oxygen saturation to guide oxygen delivery in preterm neonates for the immediate transition after birth: a 2-Center randomized controlled pilot feasibility trial. $J$ Pediatr 2016;170:73-8.

18. Collaboration TC. The Cochrane Handbook for systematic reviews of interventions version 5.1.0. Higgins JPT, and green, S, editor 2011.

19. Jansen JP, Crawford B, Bergman G, et al. Bayesian meta-analysis of multiple treatment comparisons: an introduction to mixed treatment comparisons. Value Health 2008;11:956-64.

20. Jonas DE, Wilkins TM, Bangdiwala S, et al. Findings of Bayesian Mixed Treatment Comparison Meta-Analyses: Comparison and Exploration Using Real-World Trial Data and Simulation [Internet. Rockville (MD: Agency for Healthcare Research and Quality (US), 2013.

21. Vento $M$, Cheung P-Y, Aguar M, et al. The first golden minutes of the extremely-low-gestational-age neonate: a gentle approach. Neonatology 2009;95:286-98.

22. Peleg B, Globus O, Granot M, et al. "Golden Hour" quality improvement intervention and short-term outcome among preterm infants. J Perinatol 2018;31.

23. Shah V, Hodgson K, Seshia M, et al. Golden hour management practices for infants. Paediatr Child Health 2018;23:70-6.

24. Shamseer L, Moher D, Clarke M, et al. Preferred reporting items for systematic review and meta-analysis protocols (PRISMA-P) 2015: elaboration and explanation. BMJ 2015;349.

25. Hutton B, Salanti G, Caldwell DM, et al. The PRISMA extension statement for reporting of systematic reviews incorporating network meta-analyses of health care interventions: checklist and explanations. Ann Intern Med 2015;162:777-84.

26. Papile LA, Burstein J, Burstein R, et al. Incidence and evolution of subependymal and intraventricular hemorrhage: a study of infants with birth weights less than 1,500 GM. J Pediatr 1978;92:529-34.

27. World-class systematic review management. Covidence systematic review software, veritas health innovation. Melbourne, Australia, 2019. 
28. PRISMA. Preferred Reporting Items for Systematic Reviews and Meta-Analyses [online], 2018. Available: http://www.prismastatement.org/statement.htm [Accessed 20 Apr 2018]

29. Introduction to Evidence Synthesis. Network Meta-Analysis for Decision Making [Internet. John Wiley \& Sons, Ltd, 2018: 1-17.

30. Riley RD, Higgins JPT, Deeks JJ. Interpretation of random effects meta-analyses. BMJ 2011;342.

31. Higgins JPT, Thompson SG, Spiegelhalter DJ. A re-evaluation of random-effects meta-analysis. J R Stat Soc Ser A Stat Soc 2009;172:137-59.

32. Salanti G, Ades AE, loannidis JPA. Graphical methods and numerical summaries for presenting results from multiple-treatment metaanalysis: an overview and tutorial. J Clin Epidemiol 2011;64:163-71.

33. Chaimani A, Higgins JPT, Mavridis D, et al. Graphical tools for network meta-analysis in STATA. PLoS One 2013;8:e76654.

34. Mavridis D, Welton NJ, Sutton A, et al. A selection model for accounting for publication bias in a full network meta-analysis. Stat Med 2014;33:5399-412.

35. Lambert PC, Sutton AJ, Burton PR, et al. How vague is vague? A simulation study of the impact of the use of vague prior distributions in MCMC using WinBUGS. Stat Med 2005;24:2401-28.

36. Cornell JE, Mulrow CD, Localio R, et al. Random-effects metaanalysis of inconsistent effects: a time for change. Ann Intern Med 2014;160:267-270-70.

37. DerSimonian R, Laird N. Meta-Analysis in clinical trials. Control Clin Trials 1986;7:177-88.

38. Dias S, Sutton AJ, Ades AE, et al. Evidence synthesis for decision making 2: a generalized linear modeling framework for pairwise and network meta-analysis of randomized controlled trials. Med Decis Mak 2013;33.

39. Gelman A, Carlin J, Stern H, et al. Chapter 2: Single Parameter models. In: Bayesian data analysis. 3th edn. New York: CRC Press, 2015.

40. Gabry J, Simpson D, Vehtari A, et al. Visualization in Bayesian workflow, 2017. Available: http://arxiv.org/abs/1709.01449

41. Welton NJ, Caldwell DM, Adamopoulos E, et al. Mixed treatment comparison meta-analysis of complex interventions: psychological interventions in coronary heart disease. Am J Epidemiol 2009;169:1158-65.

42. Dias S, Welton NJ, Sutton AJ, et al. Evidence synthesis for decision making 3: subgroups, meta-regression, bias, and Bias-Adjustment. Med Decis Mak [Internet] 2013;33:597-606.

43. Turner RM, Davey J, Clarke MJ, et al. Predicting the extent of heterogeneity in meta-analysis, using empirical data from the Cochrane database of systematic reviews. Int J Epidemiol 2012;41:818-27.

44. Gelman A, Rubin DB. Inference from iterative simulation using multiple sequences. Statistical Science 1992;7:457-72.

45. Plummer M. JAGS: just another Gibbs sampler, 2016. Available: http://mcmc-jags.sourceforge.net/ [Accessed 14 Mar 2017].
46. R Core Team. R: a language and environment for statistical computing, 2018. Available: https://cran.r-project.org/

47. Dias S, Welton NJ, Sutton AJ, et al. Evidence synthesis for decision making 4: inconsistency in networks of evidence based on randomized controlled trials. Med Decis Making 2013;33:641-56.

48. Jackson D, Barrett JK, Rice S, et al. A design-by-treatment interaction model for network meta-analysis with random inconsistency effects. Stat Med 2014;33:3639-54

49. Rhodes KM, Turner RM, Higgins JPT. Predictive distributions were developed for the extent of heterogeneity in meta-analyses of continuous outcome data. J Clin Epidemiol 2015;68:52-60.

50. Guyatt G, Oxman AD, Akl EA, et al. Grade guidelines: 1. IntroductionGRADE evidence profiles and summary of findings tables. J Clin Epidemiol 2011;64:383-94.

51. Balshem H, Helfand M, Schünemann HJ, et al. Grade guidelines: 3 . rating the quality of evidence. J Clin Epidemiol 2011;64:401-6.

52. Guyatt $\mathrm{GH}$, Oxman AD, Vist G, et al. GRADE guidelines: 4. Rating the quality of evidence--study limitations (risk of bias). J Clin Epidemiol 2011;64:407-15.

53. Guyatt $\mathrm{GH}$, Oxman AD, Kunz R, et al. GRADE guidelines 6. Rating the quality of evidence--imprecision. J Clin Epidemiol 2011;64:1283-93.

54. Guyatt GH, Oxman AD, Kunz R, et al. GRADE guidelines: 7. Rating the quality of evidence--inconsistency. J Clin Epidemiol 2011;64:1294-302.

55. Guyatt GH, Oxman AD, Montori V, et al. GRADE guidelines: 5 . Rating the quality of evidence--publication bias. J Clin Epidemiol 2011;64:1277-82.

56. Donegan S, Williamson P, Gamble C, et al. Indirect comparisons: a review of reporting and methodological quality. PLoS One 2010;5:e11054.

57. Baker SG, Kramer BS. The transitive fallacy for randomized trials: if a bests $\mathrm{B}$ and $\mathrm{B}$ bests $\mathrm{C}$ in separate trials, is a better than $\mathrm{C}$ ? BMC Med Res Methodol 2002;2.

58. Cipriani A, Higgins JP, Geddes JR, et al. Conceptual and technical challenges in network meta-analysis. Annals of internal medicine 2013;159:130-7.

59. Puhan MA, Schünemann HJ, Murad MH, et al. A grade Working group approach for rating the quality of treatment effect estimates from network meta-analysis. BMJ 2014;349.

60. van Valkenhoef G, Tervonen T, Zhao J, et al. Multicriteria Benefitrisk assessment using network meta-analysis. J Clin Epidemiol 2012;65:394-403.

61. Dias S, Welton NJ, Sutton AJ, et al. Evidence synthesis for decision making 5: the baseline natural history model. Med Decis Mak 2013;33:657-70.

62. Tervonen T. New directions in stochastic Multicriteria acceptability analysis, 2007. Available: http://www.utupub.fi/handle/10024/28151 [Accessed 11 Nov 2018]. 